\section{Degeneration vs. degeneracy: a neural systems approach to functional reorganization}

\section{Valenzuela, P Sachdev, M Breakspear}

School of Psychiatry UNSW, Sydney, Australia

Background: The term functional reorganization has been used to describe a number of dynamic neural processes related to changes in brain-behaviour connectivity. These include process efficiency, such that localized metabolic work decreases with expertise; process redundancy, whereby subtly different neural networks are able to resolve a given computational problem; and degeneracy, in which novel brain-behaviour relationships can be expressed in response to lesion or challenge. These processes are argued to reside within the framework of a type of 'neural Darwinism': dynamic brain-behaviour relations are in a constant state of competition for the most effective resolution of a given computational task, with selection pressure at the level of metabolic economy.

Methods: Exemplars of process efficiency, redundancy and degeneracy will be presented from a functional neuroimaging study of working memory and association learning processes in both healthy older subjects and those with mild cognitive impairment.

Results: Individuals with either no neurological deficit or incipient Alzheimer's disease (as indicated by medial temporal lobe atrophy) show a large degree of variance in response to increasingly difficult working memory tasks, as assessed by a multivariate analysis of brain-behaviour correlations.

Conclusions: The principles of efficiency, redundancy and degeneracy are applicable to the functional response to any static or progressive brain lesion. Novel approaches to describing and quantifying these processes may therefore improve our understanding of the clinicopathological disconnect observed in a number of clinical areas, in particular neurodegeneration.

\section{Modeling risk from conception to disease: issues in the design of a population health database for psychiatric research}

\section{G Valuri, M Croft, V Morgan, A Jablensky}

School of Psychiatry and Clinical Neurosciences, University of Western Australia, Perth, Australia

Background: In Western Australia, mental health register data are linked to other state-wide health registers.
A population-based study of children born in Western Australia, 1980-2001, to mothers with schizophrenia or affective psychosis and mothers with no psychiatric illness aimed to examine genetic and environmental risk factors for schizophrenia. Longitudinal linked data were used to analyze health patterns and associations of these children.

Methods: The linked data were of such diverse types, it was difficult to retrieve data for analysis based on the relationships between individuals. A data model was required to manage this complexity. We describe the database design and issues in its construction and the adaptation of a validated system, which uses case note reviews for scoring obstetric complications as risk factors for psychiatric morbidity, for use with electronic registers.

Results: The database includes 472733 births to 249119 women, with paternal data available for most children. The data model design views all records as related to a person with one or potentially many 'events' recorded across health registers over the study period, and linked by a unique identifier. It allows for consideration of families from an intergenerational perspective. We can easily extract information such as the number of siblings in the database, family sizes, intervals between siblings' births, illnesses within families and changes of residences between siblings' births.

Conclusion: A data model has been implemented that permits intergenerational gene-environment analysis and expansion to include new data and validation of event records across datasets.

\section{Integrating vocational services into Australian community mental health services}

\section{G Waghorn}

Queensland Centre for Mental Health Research (QCMHR), Brisbane, Australia

Integrating vocational services into community mental health teams is now recognized as an evidence-based practice in psychiatric vocational rehabilitation. This presentation will summarize the evidence supporting service integration and outline progress at several Australian demonstration sites. This is proving to be a challenging enterprise because of organizational cultural differences, and structural and systemic barriers. Nevertheless, the results obtained to date indicate that integration is not only feasible, but necessary, given the potential benefits and low additional costs once an integrated approach is established. 